\title{
Detection of Brain Tumour in MRI Scanned Images using DWT and SVM
}

\author{
B. Shoban $\mathrm{Babu}^{\# 1}$ and S. Varadarajan $*^{2}$ \\ ${ }^{1}$ Associate Professor, Department of ECE, SVCET, RVS. Nagar, Chittoor - 517127, A.P, India \\ bshobanbabu@gmail.com \\ ${ }^{2}$ Professor, Department of ECE, SVUCE, SVU, Tirupati - 517502, A.P, India \\ varadasouri@gmail.com
}

\begin{abstract}
-detection of the tumour in a human brain is a challenging problem, due to the arrangement of the tumour cells in the brain. This paper presents an analytical method that improves the detection of brain tumour cells in its early stages and to analyze anatomical structures by classification of the samples in support vector machine and tumour cell segmentation of the sample using gray level co-occurrence matrix and extracted features. The support vector machine is used to train and classify the stage of brain tumour that would be benign, malignant or normal, and classifying the tumour for $80 \%$. The automatic support intelligent system is proposed to detect brain tumour through the combination of discrete wavelet transform and support vector machine. This system helps to diagnose brain tumour and in vain for its treatment.
\end{abstract}

Keywords-Discrete Wavelet Transform (DWT), Support Vector Machine (SVM), Gray Level Cooccurrence Matrix (GLCM), Power Constraint Contrast Enhancement (PCCE)

\section{INTRODUCTION}

An intracranial mass of abnormal cells in the brain that have grown out of control is referred to as brain tumour. These uncontrolled expansion of irregular cells are termed as primary, if originates in the brain and secondary, if originates in other parts of the body and spread to the brain through metastasis. Primary brain tumours can be benign (non-cancerous) or malignant (cancerous), whereas secondary brain tumours are malignant. Both primary and secondary tumour are dangerous and could prove fatal if not detected in early stage. As the space inside the skull is very limited, the growth of tumour inside the skull could increase the intracranial pressure causing edema, reduced blood flow, displacement and degeneration of other tissues that control important body functions. Survival rates of the affected individual with brain tumours vary widely, depending on the type of tumour, however for brain tumour prognosis and successful treatment, therapy planning, early and accurate tumour diagnosis is imperative [1].

Medical imaging is becoming a very important aspect in clinical applications from diagnosis to treatment. Magnetic Resonance Imaging (MRI) is a very important diagnostic tool for analysis of human soft tissue. Magnetic resonance imaging is popularly used in diagnosis of brain tumour because, it is a non-invasive technique. However the diagnosis is inherently challenging due to the large variance and complexity of tumour characterization in images, such as size, structure, location and intensities and can only be performed by professional radiologists. MRI scanned images may contain noise that could pave path to inaccuracies in classification [2].

Magnetic resonance imaging (MRI) is an imaging technique that produces high quality images of the atomical structures of the human body, especially in the brain, and provides rich information for clinical diagnosis and biomedical research. The diagnostic values of MRI are greatly magnif ed by the automated and accurate classif cation of the MRI images. Discrete Wavelet transform is an e ectiv e tool for feature extraction from MR brain images, because it allows analysis of images at various levels of resolution due to its multiresolution analytic property. However, this technique requires large storage and is computationally expensive $[3-4]$.

\section{PRoposed METHOD}

This paper deals with detection of brain tumour using discrete wavelet transform and SVM classifier from MR images of brain. The organization of the paper is as follows. In this section proposed algorithm has been described. The methodology used for implementing the proposed technique of brain tumour detection has been provided in section III. The results attained have been discussed in section IV, and finally Section V presents conclusion. 


\section{A. Discrete Wavelet Transform}

Discrete Wavelet Transform, a basic wavelet transform uses two filter banks, one is analysis filter bank that transforms noised test image into transform coefficients and another one is synthesis filter bank that does the inverse operation i.e. transforms transform coefficients into estimated image [5-6].

Analysis filter bank of discrete wavelet transform shown by figure 1 consists of Low Pass Filter (LPF), High Pass Filter (HPF) and down-sampling factor of $(2,2)$. In level-1, the noised test image of size say $512 \times 512$ has been filtered and decomposed into four sub-bands with each band of size say 256 X 256 image. In level-2 transform coefficients of LL sub-band are filtered again and downsampled by factor of $(2,2)$ and decomposed again into four sub-bands, with each band of size say 128 X 128 image, this decomposition has been performed until the coarse transform coefficients are obtained. In the similar way, after fifth level of decomposition noised test image of size say 512 X 512 image has been decomposed into transform coefficients of size say 16 X 16 .

Synthesis filter bank of discrete wavelet transform inverse of analysis filter bank of discrete wavelet transform shown by figure 2 reconstructs the estimated image with the help of low pass filter, high

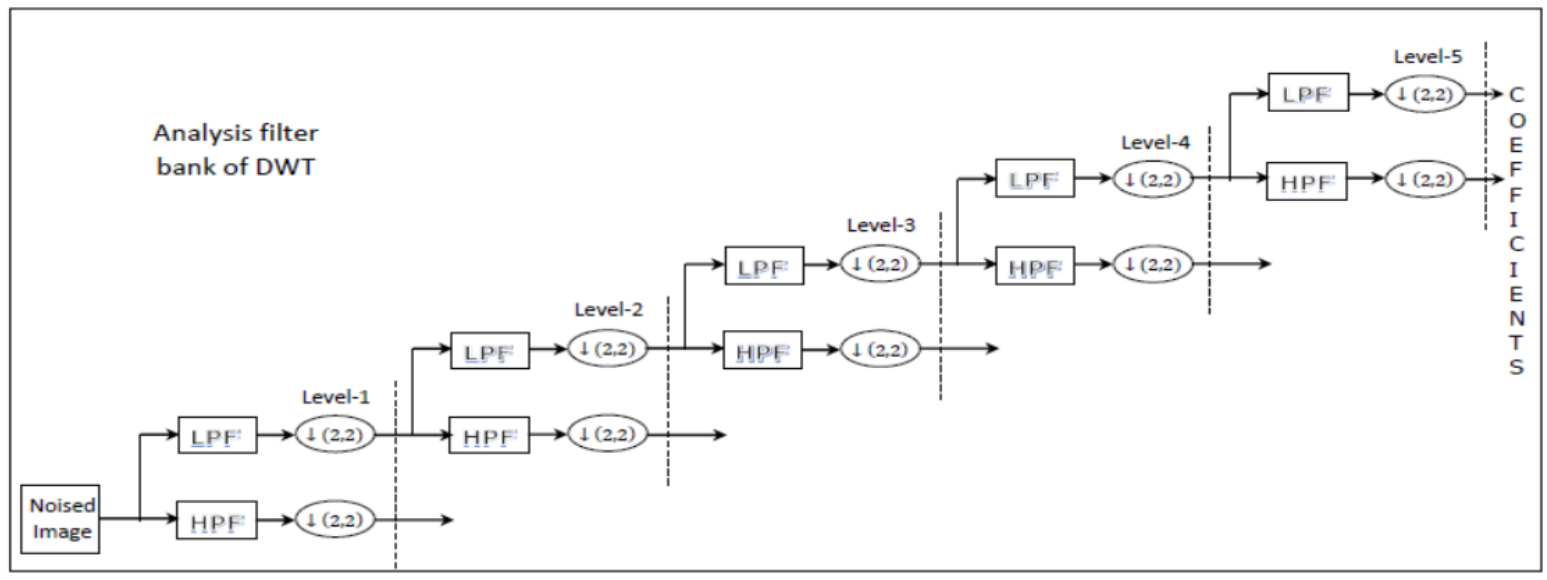

Figure 2. Analysis filter bank of DWT

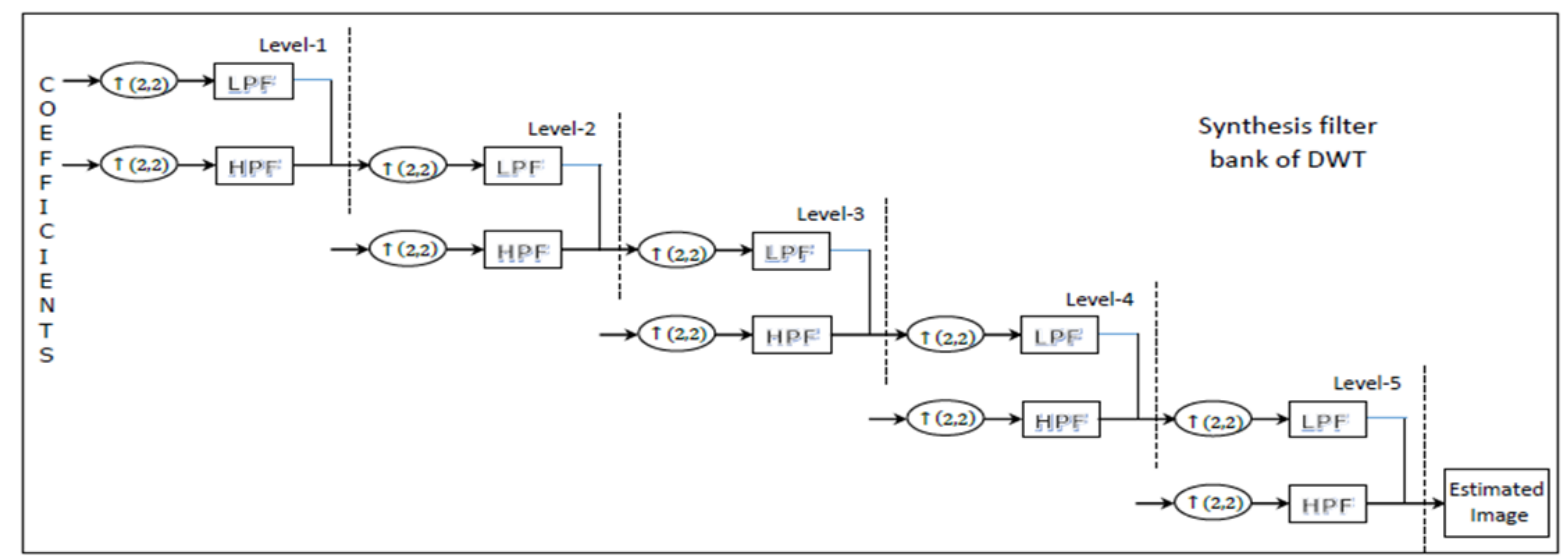

Figure 2. Synthesis filter bank of DWT

pass filter, and up-sampling factor of $(2,2)$. In level-1 the transform coefficients after five level decomposition of size say 16 X 16 obtained from analysis filter bank of discrete wavelet transform have been up-sampled by a factor of $(2,2)$, filtered using low pass filter and high pass filter, and convolved into coefficients of size say 32 X 32. In level-2 these coefficients have been again up-sampled by a factor of $(2,2)$, then filtered and convolved into coefficients of size say 64 X 64 . In consequence, after level-5 i.e. fifth level of convolution, coarse transform coefficients obtained from analysis filter bank of discrete wavelet transform of size say 16 X 16 image has been reconstructed into an estimated image of size say 512 X 512.

\section{B. Segmentation}

The segmentation is based on thresholds, morphological operations and modelled by gaussian functions. Usually MRI images have non-isotropic voxel sizes; in order to get isotropic image compute the threshold by considering the different tissues gray levels and the image histogram as a probability density function of the image gray levels figured by equation 1 . 


$$
P(i)=n_{i} / N
$$

Where $n_{i}=$ Number of voxels with gray level with $i=\{0,1,2,3 \ldots \ldots w-1\}$

$N=$ Number of voxels in image,

$P(i)=$ Probability for a voxel to get intensities

Flowchart shown in figure 3 outlines the path for detection of brain tumour using discrete wavelet transform and SVM classifier from MR images of brain for segmentation MR images of the human brain and segmentation of pre-processed image MR image has been accomplished in the following three steps

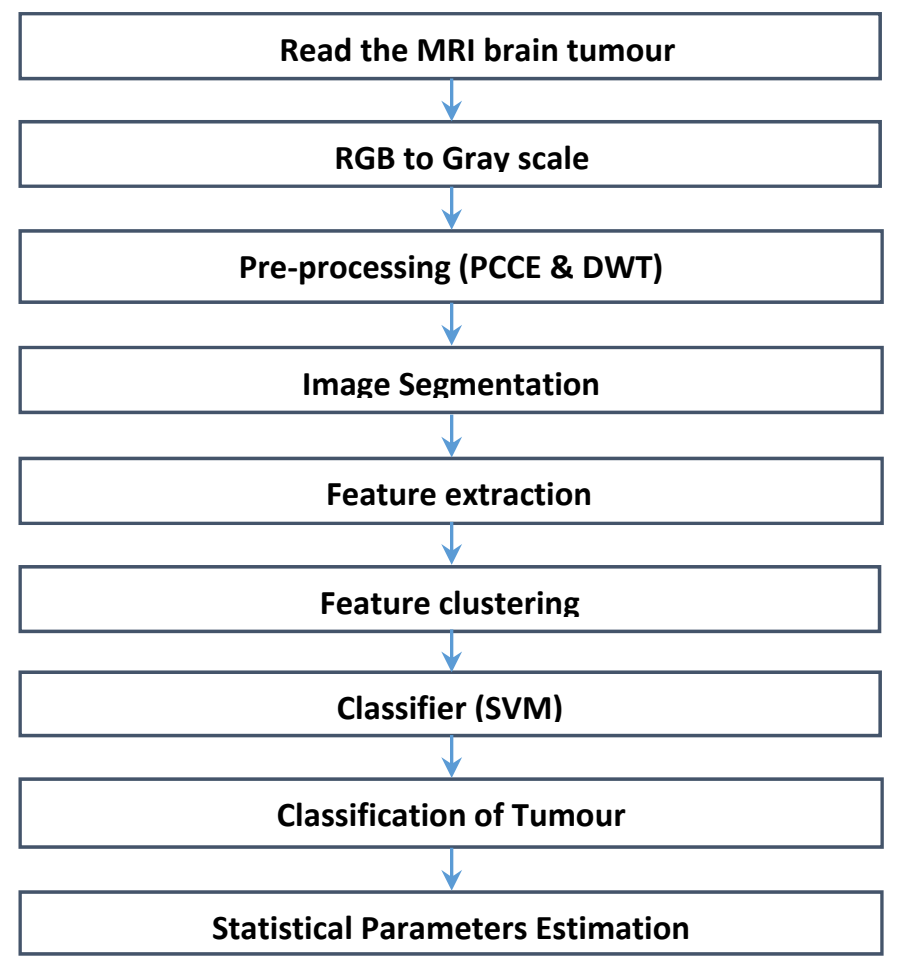

Figure 3. Flowchart

\section{1) Background elimination}

The other method is used to find the threshold, $T_{\text {otsu }}$ that limits the inside class variance between two classes, for our situation, one class is shaped by the low power voxels comparing to air, bone and part of the Cerebro Spinal Fluid (CSF) (background) and other class is composed of the other tissues including the gray matter (background) and white matter of the brain (foreground).the with in class variance is defined equation 2.

$$
\sigma_{\text {within }}^{2}\left(T_{\text {otsu }}\right)=n_{B}\left(T_{\text {otsu }}\right) \sigma_{B}^{2}\left(T_{\text {otsu }}\right)+n_{F}\left(T_{\text {otsu }}\right) \sigma_{F}^{2}\left(T_{\text {otsu }}\right)
$$

Where $\sigma_{B}^{2}\left(T_{\text {otsu }}\right)$ and $n_{B}\left(T_{\text {otsu }}\right)$ are variance and number of Voxels in the background $\sigma_{F}^{2}\left(T_{o t s u}\right)$ and $n_{F}\left(T_{o t s u}\right)$ are variance and number of Voxels in the foreground.

\section{2) Brain Identification}

The brain is first separated from other tissues by applying threshold, $T_{S}$ based on an image histogram .Threshold for separating the brain is defined by an equation 3 .

$$
T_{S}=T_{o t s u}+\gamma\left(\mu_{g m}-T_{o t s u}\right)
$$

Where $\mu_{g m}=$ estimation of the mean gray level of the $g_{m}$, which corresponds to the highest value in the histogram of the image.

In this method an empirical threshold of 0.08 is used and the condition shown in equation 4 should be satisfied to accept the tissue removal.

$$
\frac{v_{m 2}^{\text {out }}}{v_{m}}<0.08
$$

Here $v_{m 2}^{\text {out }}=$ Mask laying outside the model image (light gray)

$v_{m}=$ Dark gray 
After the procedure described above some parts of other tissues, such as dura, still remain around the brain.so other thresholds are required which are formed by Gaussian approximation of the image histogram.

The approximated histogram shown by equation 5 is constructed, by modelling these classes with Gaussian and by applying this model to the MR image we will get the tumour separately from the MRI brain tumour image.

(i) Background noise -it may form a peak in the histogram but often does not

(ii) Gray matter-forms the central peak in the histogram

(iii) White matter - it forms the peak at the right side of the histogram and

And other tissues with high gray value

$$
P(i: v)=\sum_{k=1}^{3} P_{k} \exp \left[-\frac{1}{2}\left(\frac{i-\mu_{k}}{\sigma_{k}}\right)^{2}\right]
$$

Where $i=$ Gray level,

$\boldsymbol{\mu}_{\boldsymbol{k}}=$ Mean of gray level k

$P_{k}=$ Probability for a voxel of class k to obtain intensity $\mu_{k}$,

$\sigma_{k}=$ Standard deviation of the Gaussian function.

\section{Support Vector Machine classifier}

SVM classifier is the most efficient algorithm, which utilize the concept of kernel substitution and are known as kernel methods. The training set of instance-label pairs are given as $\left(x_{i}, y_{i}\right) ; i=1 \ldots l$ where $x_{i} \in R^{n}$ and $y \in\{1,-1\}^{l}$, the Support Vector Machines (SVM) [7-9] require the solution of the optimization problem, i.e., the SVM intends to minimize an error function given in equation (6) with the constraint shown in equation (7)

$$
\begin{aligned}
& \min _{w, b, \xi} \frac{1}{2} \mathcal{W}^{T}+\mathcal{C} \sum_{i=1}^{l} \xi_{i} \ldots \ldots \ldots \ldots \ldots \ldots \ldots \ldots \ldots \ldots \ldots \ldots \\
& y_{i}\left(w^{T} \phi\left(x_{i}\right)+b\right) \geq 1-\xi_{i} \quad \xi_{i} \geq 0
\end{aligned}
$$

The training vectors $x_{i}$ is mapped into a higher dimensional space by the function $\phi$ and subsequently SVM finds a linear separating hyper-plane with the maximal margin in this higher dimensional space. $c>0$, is the penalty parameter of the error term. Also $K\left(x_{i}, x_{j}\right) \equiv \phi\left(x_{i}\right)^{T} \phi\left(x_{j}\right)$ is known as the kernel function. By reducing the error function, the SVM learns the extracted feature set $x_{i}$ effectively in order to categorize the normal or abnormal that are analogous to the training set. From the training data, the SVM classifier learns about the class in which the normal or tumour is present. Once the SVM is trained, it can classify any brain MR image dataset in the similar manner. In the classification phase, the selected features that are used in the training process to train the SVM classifier are extracted for testing the brain MRI image. The features set is given to the trained SVM for classifying the given brain MR image [10-12]. In this paper three types of SVM classifiers have been used to classify the resultant image and finding out the accuracy of the input MRI image to say linear kernel, RBF kernel and Polynomial kernel.

\section{METHODOLOGY}

From the training gene data, the SVM learns well about the class in which the normal or tumour is present. Once the SVM is trained appropriately, it achieves the capability to classify any brain MR image collected in dataset in the similar fashion.

In the classification phase, all the features that are used in the training process are extracted for testing the tumour image in a similar manner and subsequently the clustering of the features is performed and the SVMtesting process is carried out using the extracted feature set combination. The features set is given to the trained SVM and therefore the class of the given MRI brain image is obtained efficiently.

\section{RESULTS AND DiSCUSSIONS}

The performance of the proposed methodology has been evaluated using Discrete wavelet Transform and SVM classifier and programed using MATLAB R2014a by exploring its wavelet tool box, image processing and simulation toolboxes. The results have been discussed in the form of following steps.

- $\quad$ PSNR using DWT $52.5159 \mathrm{~dB}$ and it has been observed that PSNR has been increased by $11.16 \mathrm{~dB}$ using PCCE [13], and its screen shot is shown in the figure 4 and figure 5 respectively.

- Tumour present in the test image has been identified using Feature extraction and feature clustering techniques. 
- Using GLCM and SVM classifier the tumour present in the test image has been classified as benign (noncancerous) or malignant (cancerous).

- Accuracy of the proposed methodology has been obtained as $80 \%$ and respective screen shot Shown in the figure 6.

- $\quad$ Figure 7 shows histograms and 3D plots of DWT and PCCE.

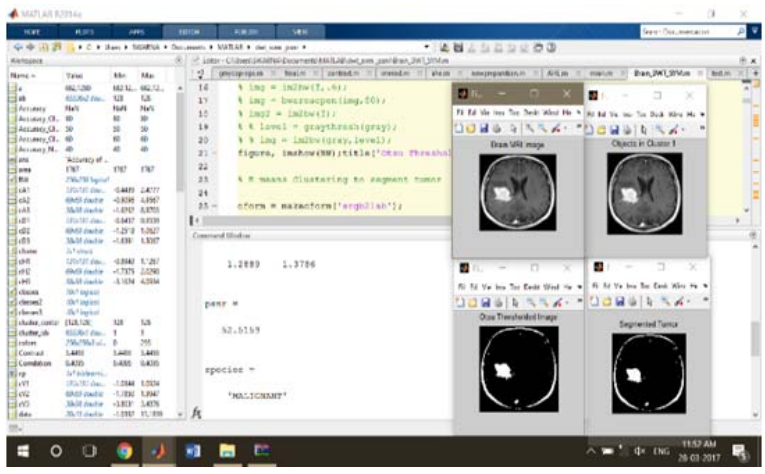

Figure 4. Screen shot of DWT (PSNR)

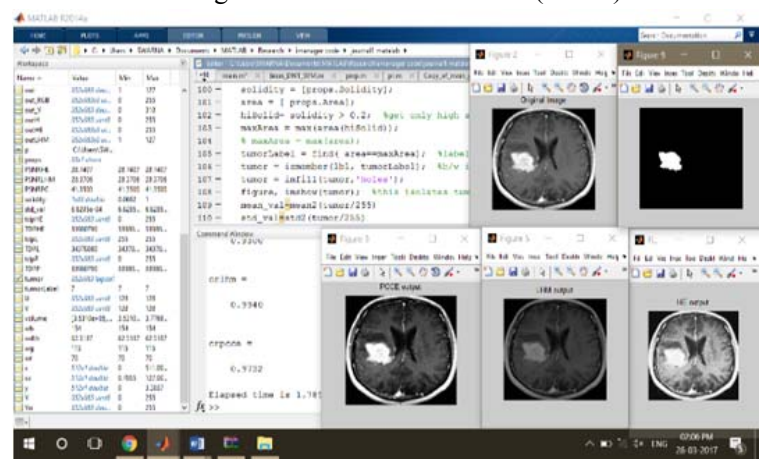

Figure 5. Screen shot of PCCE (PSNR)

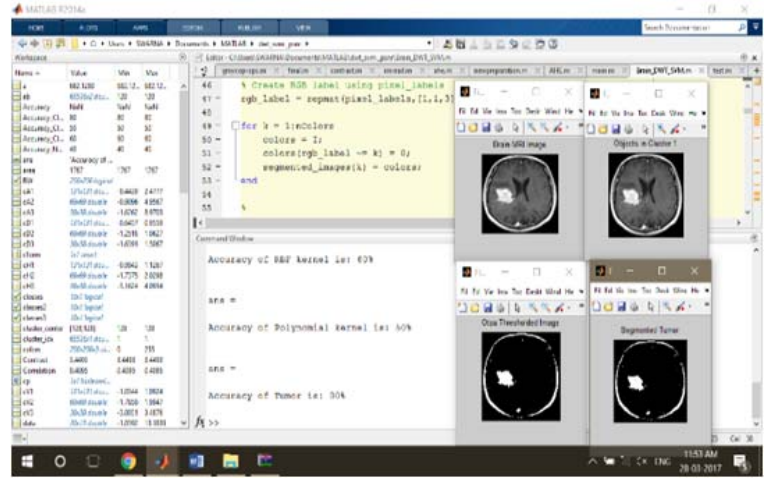

Figure 6. Screen shot of SVM classifier

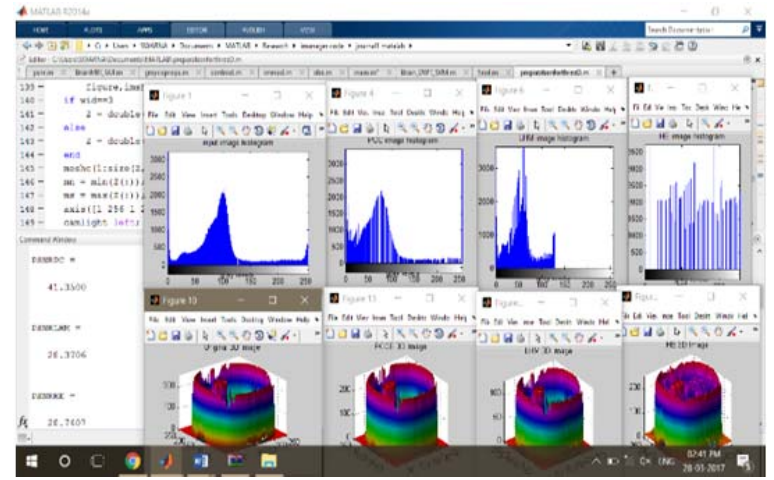

Figure 7. Screen shot of Histogram and 3D image for DWT. 


\section{CONCLUSion AND Future SCOPE}

This paper presents an efficient method of classifying MRI brain images into normal and abnormal Tumour, using a SVM. This paper presents a method called Discrete wavelet transform (DWT) features is extracted from the brain MRI images, which signify the important texture features of Tumour tissue and gives very auspicious results in classifying MR images. From the experimental results, it is observed that SVM shows a classification accuracy of $80 \%$, and demonstrated that the suggested feature method performs well and achieved good recognition results for Tumour classification. It has been observed from the experiments that the system could not distinguish Astrocytomas class with high accuracy and is of future interest.

\section{REFERENCES}

[1] Zhang Y, Wu L and Wang S, "Magnetic resonance brain image classif cation by an improved artif cial bee colony algorithm," Progress In Electromagnetics Research, Vol. 11, No. 6, pp. 65-79, 2011.

[2] Golestanirad L, Izquierdo A P, Graham S J, Mosig J R, and Pollo C, "Effect of realistic modeling of deep brain stimulation on the prediction of volume of activated tissue," Progress In Electromagnetics Research, Vol. 126, pp. 1-16, 2012.

[3] Scapaticci R, Di Donato L, Catapano I, and Crocco L, "A feasibility study on microwave imaging for brain stroke monitoring," Progress In Electromagnetics Research B, Vol. 40, pp. 305-324, 2012.

[4] Swarnalatha S, and Sathyanarayana P, "Denoising of Images by means of Wavelets and Contourlets using Bi-shrink filter", i-manager's Journal on Image Processing, vol. 3, no. 3, pp. 11-16, July - September 2016.

[5] Emin Tagluk M, Akin M and Sezgin N, "Classif cation of sleep apnea by using wavelet transform and artif cial neural networks," Expert Systems with Applications, Vol. 37, No. 2, pp. 1600-1607, 2010.

[6] Ananda Resmi S and Thomas T, "Texture Description of low grade and high grade Glioma using Statistical features in Brain MRI", International Journal of Recent Trends in Engineering and Technology, Vol. 4, No. 3, Nov 2010, pp.27-33

[7] Albregtsen F, "Statistical Texture Measures Computed from Gray Level Co-occurrence Matrices", Image Processing Laboratory, Department of Informatics, University of Oslo, 2008.

[8] Dong M, Choi K and Zhong L, "Power modeling of graphical user interfaces on OLED displays," in Proc. Des. Autom. Conf.., pp. 652657, Jul. 2009

[9] Fritz Albregtsen, "Statistical Texture Measures Computed from Gray Level Co-occurrence Matrices," Image Processing Laboratory, Department of Informatics, University of Oslo, November 5, 2008.

[10] Boser B E, Guyon I and Vapnik V, "A training algorithm for optimal margin classifiers", In Proc. of the Fifth Annual Workshop on Computational Learning Theory, ACM Press, 1992, pp. 144-152.

[11] Cortes C and Vapnik V, "Support-vector network", Machine Learning, vol. 20, pp. 273-297, 1995.

[12] Neha Tirpude and Rashmi Welekar, "Automated Detection and Extraction of Brain Tumour from MRI Images", International Journal of Computer Applications (0975 - 8887) Volume 77- No.4, pp. 26-30. September 2013.

[13] Shoban Babu B, Varadarajan S and Swarnalatha S, "Contrast Enhancement Based Brain Tumour MRI Image Segmentation And Detection With Low Power Consumption ”, i-manager's Journal of Image Processing Vol. 11, No, 3 No. 2 April - June 2016.

\section{AUTHOR PROFILE}

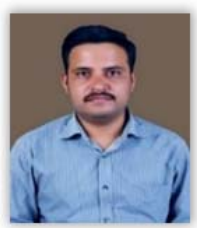

B. Shoban Babu

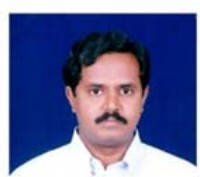

Dr. S. Varadarajan received bachelor's degree in ECE domain from SVUCE in 1987, master's degree from KU warangal in 1993, and doctor's degree from SVUCE in 2005. Served as an Assistant professor at NIT Warangal (Sep. 92 - Nov. 92), as Assistant professor and Associate professor (1992-2013) and as professor from 2013 at SVUCE, Tirupati. His research is focused on, Wireless Communications, Signal and Image Processing. At presently chairing the secretary, APSCHE, Hyderabad.

Dr. S. Varadarajan 